\title{
RIEMANN SPACE WITH TWO-PARAMETRIC HOMOGENEOUS HOLONOMY GROUP
}

\author{
MINORU KURITA
}

The rotational part of the holonomy group of a Riemann space is called its homogeneous holonomy group. A Riemann space, whose homogeneous holonomy group is one-parametric, was investigated by Liber and an alternative treatment of the same problem was given by S. Sasaki [1]. I will treat here a Riemann space with two-parametric homogeneous holonomy group and prove the following theorem by the method analogous to that of [1]. I thank Prof. T. Ootuki for his kind advice.

THEOREM. If the homogeneous holonomy group of an n-dimensional Riemann space is two-parametric, the space is a direct sum of two non-flat two-dimensional Riemann spaces and a n-4-dimensional flat space; namely the line-element of our space is given by

$$
d s^{2}=d \sigma_{1}^{2}+d \sigma_{2}^{2}+\sum_{i=5}^{n}\left(d x^{i}\right)^{2}
$$

where

$$
d \sigma_{i}=\sum_{i, j=1,2} g_{i j}\left(x^{1}, x^{2}\right) d x^{i} d x^{j}, \quad d \sigma_{i}=\sum_{i, j=3,4} g_{i j}\left(x^{3}, x^{4}\right) d x^{i} d x^{j}
$$

0. Let the line element of a general Riemann space be given by

$$
d s^{2}=\sum_{i=1}^{n}\left(\omega_{i}\right)^{2} .
$$

If we take a rectangular frame in the tangent euclidean space at any point of the Riemann space, a Riemannian connection is given by

$$
d \mathbf{A}=\omega_{i} \mathbf{e}_{i}, \quad d \mathbf{e}_{i}=\omega_{i j} \mathbf{e}_{j} \quad\left(\omega_{i j}=-\omega_{j i}\right) .
$$

If we denote the outer derivative of $\omega$ by $d \omega$, we have

$$
d \omega_{i}=\left[\omega_{j} \omega_{j i}\right] .
$$

Taking the outer derivative of both sides, we get

$$
\left[\omega_{j} \prod_{j i}\right]=0
$$

where $I_{i j}$ is given by

Received September 18, 1951. 


$$
\Pi_{i j}=d \omega_{i j}-\left[\omega_{i k} \omega_{k j} .\right] .
$$

In general we define a product of two matrices $\Omega=\left(\omega_{i p}\right)$ and $\Pi=\left(\pi_{p u}\right)$ with Pfaffians or differential forms as coefficients by a matrix

$$
[\Omega \Pi]=\left(\left[\omega_{i p} \pi p u\right]\right)
$$

and an outer derivative of $\Omega$ by a matrix

$$
d \Omega=\left(d \omega_{i p}\right) .
$$

Then putting

$$
\omega=\left(\omega_{1}, \ldots, \omega_{n}\right), \quad \Omega=\left(\omega_{i j}\right), \quad \Pi=\left(\Pi_{i j}\right),
$$

(2), (4), (3) can be written as

$$
\begin{gathered}
d \omega=[\omega \Omega], \quad \Pi=d \Omega-[\Omega \Omega], \\
{[\omega \Pi]=0 .}
\end{gathered}
$$

If we rotate a rectangular frame, a new frame is given by $\overline{\mathrm{e}}_{i}=p_{i j} \mathbf{e}_{j}$. Our Riemannian connection is for this new frame determined by

$$
\bar{\omega}=\omega P^{\prime}, \quad \bar{\Omega}=P \Omega P^{\prime}+d P \cdot P^{\prime},
$$

where $P$ is the orthogonal matrix $\left(p_{i j}\right)$. If $P$ is a constant matrix, we have

$$
\bar{\Omega}=P \Omega P^{\prime} \text {. }
$$

Nexi we state lemmas which are used repeatedly in our treatment.

Lemma 1. Let $\omega_{\alpha}(\alpha=1, \ldots, k)$ be linearly independent Pfaffians with $n$ variables $x^{1}, \ldots, x^{n}$. If there exist Pfaffians $\omega_{\alpha \beta}$ such that

$$
d \omega_{\alpha}=\left[\omega_{\beta}, \omega_{\beta \alpha}\right], \omega_{\alpha \beta}=-\omega_{i \alpha}(\alpha, \beta=1, \ldots, k),
$$

then by suitable choice of variables $y^{i}=y\left(x^{1}, \ldots, x^{n}\right)(i=1, \ldots, n), \sum_{\alpha=1}^{k}\left(\omega_{\alpha}\right)^{2}$ can be written as a quadratic differential form in $k$ variables $y^{1}, \ldots, y^{k}$, namely

$$
\sum_{\alpha=1}^{k}\left(\omega_{\alpha}\right)^{2}=g_{\alpha \beta}\left(y^{1}, \ldots, y^{k}\right) d y^{\alpha} d y^{\beta} .
$$

This is seen in E. Cartan [2] p. 296.

LEMMA 2. If the rotational part $\Omega=\left(\omega_{i j}\right)$ of our connection (1) is a direct sum of two matrices, our Riemann space is a direct sum of two Riemann spaces. In other words if $\omega_{\alpha \lambda}=0 \quad(\alpha=1, \ldots, k ; \lambda=k+1, \ldots, n)$ then by a suitable choice of coordinates

$$
\begin{aligned}
d s^{2}=g_{\alpha \beta}\left(x^{1}, \ldots, x^{k}\right) d x^{\alpha} d x^{3}+ & g_{\lambda \mu}\left(x^{k+1}, \ldots, x^{n}\right) d x^{\lambda} d x^{\mu} . \\
& (\alpha, \beta=1,2, \ldots, k ; \lambda, \mu=k+1, \ldots, n)
\end{aligned}
$$

Proof. By virtue of $\omega_{a \lambda}=0$ and (2) we have 


$$
\begin{aligned}
& d \omega_{\alpha}=\left[\omega_{\beta} \omega_{\beta \alpha}\right]+\left[\omega_{\lambda} \omega_{\lambda \alpha}\right]=\left[\omega_{\beta} \omega_{\beta \alpha}\right] \\
& d \omega_{\lambda}=\left[\omega_{\alpha} \omega_{\alpha \lambda}\right]+\left[\omega_{\mu} \omega_{\mu \lambda}\right]=\left[\omega_{\mu} \omega_{\mu \lambda}\right] .
\end{aligned}
$$

Using lemma 1. we get lemma 3.

Lemma 3, If a Riemann space $S$ is a direct sum of two Riemann spaces $S_{1}$ and $S_{2}$, the homogeneous holonomy group $g$ of $S$ is the direct product of the homogeneous holonomy group $g_{1}$ of $S_{1}$ and $g_{2}$ of $S_{2}$. Especially if $g_{1}$ and $g_{2}$ are

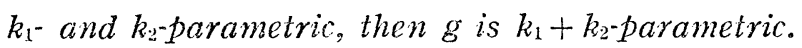

1. Now we consider a Riemann space whose homogeneous holonomy group $g$ is two-parametric. For such a space we can take rectangular frame at each point such that $Q=\left(\omega_{i j}\right)$ is an infinitesimal rotation belonging to our homogeneous holonomy group $g$. This is evident by the fundamental theorem of E. Cartan about the holonomy group. As $g$ is two-parametric, we have

$$
\Omega=\left(\omega_{i j}\right)=C_{1} \pi_{1}+C_{2} \pi_{2},
$$

where $\pi_{1}$ and $\pi_{2}$ are Pfaffians whose ratio is not a constant and $C_{1}$ and $C_{2}$ are constant skew symmetric matrices such that

$$
\left[C_{1} C_{2}\right]=C_{1} C_{2}-C_{2} C_{1}=\lambda_{1} C_{1}+\lambda_{2} C_{2} \text {. }
$$

Owing to the skewness of $C_{1}$ and $C_{2}$ two constants $\lambda_{1}$ and $\lambda_{2}$ are zero. In fact let $\lambda_{1}$ be not zero, then putting $\lambda_{1} C_{1}+\lambda_{2} C_{2}=\bar{C}_{1}$ and $C_{2} / \lambda_{1}=\bar{C}_{2}$ we have

$$
\left[\bar{C}_{1} \bar{C}_{2}\right]=\bar{C}_{1} \text {. }
$$

For an orthogonal matrix $P P \bar{C}_{1} P^{\prime}$ and $P \bar{C}_{2} P^{\prime}$ are skew symmetric matrices and moreover

$$
\left[P \overline{\mathcal{C}}_{1} P^{\prime}, P \bar{C}_{2} P^{\prime}\right]=P \bar{C}_{1} P^{\prime} .
$$

If we select $P$ suitably, we have

$$
P \vec{C}_{1} P^{\prime}=\left(\begin{array}{cccccc}
A_{1} & & & & & \\
& \cdot & & & & \\
& & A_{l} & & \\
& & 0 & & \\
& & & \cdot & \\
& & & & 0
\end{array}\right)
$$

where

$$
A_{i}=\left(\begin{array}{rr}
0 & \alpha_{i} \\
-\alpha_{i} & 0
\end{array}\right), \quad \alpha_{i} \neq 0 . \quad(i=1,2, \ldots, l) .
$$

Hence calculating the elements of the first row and the second colomn we get by (10) $\alpha_{1}=0$, which contradicts the assumption $\alpha_{1} \neq 0$. Thus we get $\lambda_{1}=0$, and similarly $\lambda_{2}=0$. So we have

$$
\left[C_{1}, C_{2}\right]=0 .
$$


Now we state one lemma.

Lemma 4. Let $C_{1}$ and $C_{2}$ be skew symmetric matrices such that $\left[C_{1}, C_{2}\right]=0$ and we assume there exists a constant $m$ for which the matrix $m C_{1}+C_{2}$ is singular. Then the Riemann space with $\Omega=\left(\omega_{i j}\right)=C_{1} \pi_{1}+C_{2} \pi_{2}$ decomposes into the direct sum of Riemann spaces.

Proof. Put $\bar{C}_{2}=m C_{1}+C_{2}$. For suitably chosen orthogonal matrix $P$ we have

$$
P \bar{C}_{2} P^{\prime}=\left(\begin{array}{cc}
A & 0 \\
0 & 0
\end{array}\right),
$$

where $A$ is non-singular. Let the corresponding formula for $P C_{1} P^{\prime}$ be

$$
P C_{1} P^{\prime}=\left(\begin{array}{ll}
K & L \\
M & N
\end{array}\right)
$$

namely $K$ and $A$ have the same number of rows and colomns. Then by the relation $\left[P C_{1} P^{\prime}, P \bar{C}_{2} P^{\prime}\right]=0$ we have $A L=0$ and $M A=0$. As $A$ is non-singular we get $L=0$ and $M=0$. Hence

$$
\begin{aligned}
\bar{\Omega} & =P \Omega P^{\prime}=P C_{1} P^{\prime} \pi_{1}+P C_{2} P^{\prime} \pi_{2} \\
& =\left(\begin{array}{cc}
K \pi_{1}+(A-m K) \pi_{2} & 0 \\
0 & N \pi_{1}-m N \pi_{2}
\end{array}\right)
\end{aligned}
$$

and by lemma 2 and (7) we get the result.

Now we treat the case when $C_{1}$ is non-singular. Then for a suitable orthogonal matrix $P$ we have

$$
P C_{1} P^{\prime}=\left(\begin{array}{lll}
\alpha_{1} Q & & \\
& \cdot \alpha_{l} Q
\end{array}\right) \text {, where } Q=\left(\begin{array}{rr}
0 & 1 \\
-1 & 0
\end{array}\right)
$$

and moreover all $\alpha_{i}>0$. Put

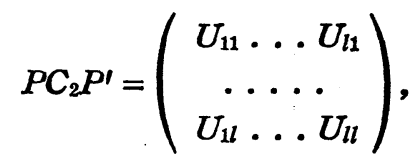

where $U_{i j}$ 's $(i, j=1,2, \ldots, l)$ are square matrices of degree 2 . Then by virtue of $\left[P C_{1} P^{\prime}, P C_{2} P^{\prime}\right]=0$ we have

$$
\alpha_{i} Q U_{i j}-U_{i j \alpha} Q=0 \quad(i \neq j),
$$

where double indices do not mean summation.

Putting $U_{i j}=\left(\begin{array}{cc}u & v \\ w & z\end{array}\right)$ we get

$$
\alpha_{j} u-\alpha_{i} z=0, \quad \alpha_{i} u-\alpha_{j} z=0, \quad \alpha_{j} v+\alpha_{i} w=0, \quad \alpha_{i} v+\alpha_{j} w=0 .
$$

As $\alpha_{i}>0, \alpha_{i} \neq \alpha_{j}$ means $\alpha_{i}^{2} \neq \alpha_{j}^{2}$ and then $u=v=w=z=0$, namely $U_{i j}=0$. For 
$\alpha_{i}=\alpha_{j}(i \neq j)$ we get $u=z$ and $w=-v$. Thus by suitable change of the order of axes of the frame the rotational part of Riemannian connection $\left(\omega_{i j}\right)$ decomposes into a direct sum of the matrices of the form

where

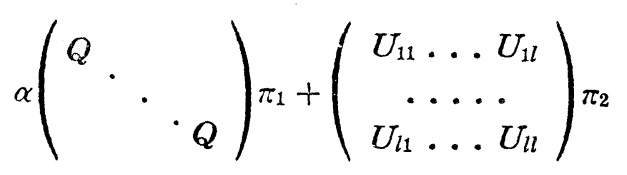

$$
U_{i j}=\left(\begin{array}{cc}
u_{i j} & v_{i j} \\
-v_{i j} & u_{i j}
\end{array}\right) \quad(i, j=1,2, \ldots, l)
$$

Now we take $\pi_{1}$ for $\alpha \pi_{1}$ and put

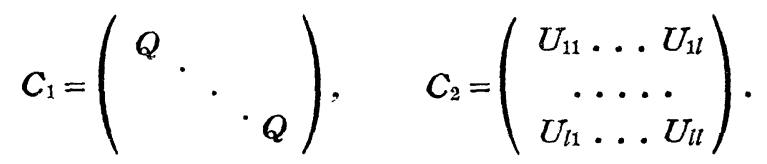

If $U_{12}, \ldots, U_{1 l}$ are all zero matrices, our Riemann space decomposes by virtue of $\Omega=C_{1} \pi_{1}+C_{2} \pi_{2}$ and lemma 2. Hence if we treat an indecomposable space, we can assume without loss of generality $U_{12}$ is not zero matrix. We put $U_{12}=\left(\begin{array}{cc}u & v \\ -v & u\end{array}\right)$ and take $C_{2} / \sqrt{u^{2}+v^{2}}$ for $C_{2}$. Then we can assume $u^{2}+v^{2}=1$. Now we transform $C_{1}$ and $C_{2}$ by an orthogonal matrix

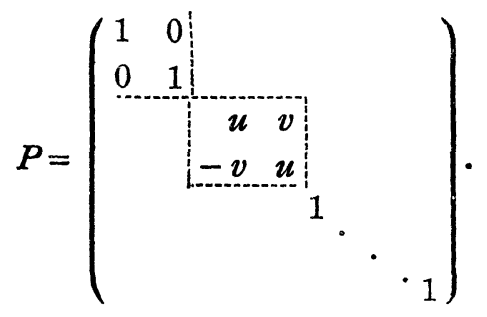

Then $P C_{1} P^{\prime}=C_{1}$, and taking $P C_{2} P^{\prime}-q C_{1}$ for $C_{2}$ where $U_{11}=\left(\begin{array}{cc}0 & q \\ -q & 0\end{array}\right)$ we get the reduced formulas

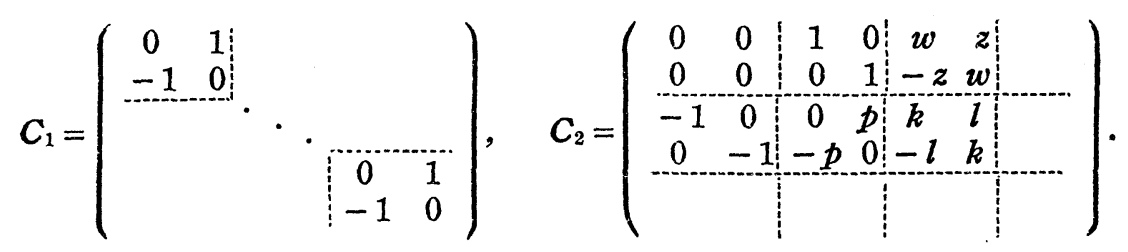

Moreover we can assume

$$
\text { det. }\left|m C_{1}+C_{2}\right| \neq 0 \text {, }
$$

because in the alternative case our space decomposes by lemma 4 . 
2. Now we investigate the space for which

$$
\Omega=C_{1} \pi_{1}+C_{2} \pi_{2},
$$

where $C_{1}$ and $C_{2}$ are given by (12) satisfying the relation (13). We get by (5)

$$
\Pi=d \Omega-[\Omega \Omega]=C_{1} d \pi_{1}+C_{2} d \pi_{2}-\left[C_{1} C_{2}\right]\left[\pi_{1} \pi_{2}\right],
$$

which by (11) reduces to

$$
\Pi=C_{1} d \pi_{1}+C_{2} d \pi_{2}
$$

Hence we have by (6)

$$
\left[\omega C_{1}, d \pi_{1}\right]+\left[\omega C_{2}, d \pi_{2}\right]=0 .
$$

Here we state one more lemma, which is evident.

Lemma 5. Let $\omega_{1}, \ldots, \omega_{n}$ be linearly independent Pfaffians and let

$$
\Omega_{1}=\frac{1}{2} a_{i j}\left[\omega_{i} \omega_{j}\right], \Omega_{2}=\frac{1}{2} b_{i j}\left[\omega_{i} \omega_{j}\right] \quad\left(a_{i j}=-a_{j i}, b_{i j}=-b_{j i}\right)
$$

and moreover

then we have

$$
\left[\omega_{1} \Omega_{1}\right]+\left[\omega_{2} \Omega_{2}\right]=0,
$$

and

$$
a_{i j}=0, \quad b_{i j}=0 \quad(i \neq 1,2 ; j \neq 1,2)
$$

$$
a_{2 i}=b_{1 i}(i=3,4, \ldots, n) .
$$

Now by (12) we have

where

$$
\begin{aligned}
& \omega C_{1}=\left(-\omega_{2}, \omega_{1},-\omega_{4} \omega_{3}, \ldots\right), \\
& \omega C_{2}=(-\rho, \tau,-\xi, \eta, \ldots),
\end{aligned}
$$

$$
\begin{aligned}
& \rho=\omega_{3}+w \omega_{5}+z \omega_{6}+\ldots \\
& \tau=-\omega_{4}+z \omega_{5}-w \omega_{6}+\ldots \\
& \xi=-\omega_{1}+p \omega_{4}+k \omega_{5}+l \omega_{6}+\ldots \\
& \eta=\omega_{2}+p \omega_{3}+l \omega_{5}-k \omega_{6}+\ldots .
\end{aligned}
$$

So by virtue of (15)

$$
\begin{aligned}
& {\left[\omega_{2} d \pi_{1}\right]+\left[\rho d \pi_{2}\right]=0,\left[\omega_{1} d \pi_{1}\right]+\left[\tau d \pi_{2}\right]=0} \\
& {\left[\omega_{1} d \pi_{1}\right]+\left[\xi d \pi_{2}\right]=0,\left[\omega_{3} d \pi_{1}\right]+\left[\eta d \pi_{2}\right]=0 .}
\end{aligned}
$$

By (16) we can take $\omega_{1}, \omega_{2}, \rho, \tau, \omega_{5}, \omega_{6}, \ldots$ as base. Then by virtue of (17) and lemma $5 d \pi_{1}$ and $d \pi_{2}$ can not have terms containing $\omega_{5}, \omega_{6}, \ldots$, and these are of the form

$$
A\left[\omega_{1} \omega_{2}\right]+B\left[\omega_{1} \rho\right]+C\left[\omega_{2} \tau\right]+D[\rho \tau] .
$$

On the other hand by (18) $d \pi_{1}$ and $d \pi_{2}$ are represented by $\omega_{3}, \omega_{4}, \xi, \eta$ only, and 
so by $\omega_{3}, \omega_{1}, \hat{s}^{\prime}=\hat{s}-p \omega_{4}, \eta^{\prime}=\eta-p \omega_{i}$. Now by (16)

$$
\begin{aligned}
& \rho=\omega_{3}+w \omega_{5}+z \omega_{6}+\ldots \\
& \tau=-\omega_{4}+z \omega_{5}-w \omega_{6}+\ldots \\
& \omega_{1}=-\xi^{\prime}+k \omega_{5}+l \omega_{6}+\ldots \\
& \omega_{2}=\eta^{\prime}-l \omega_{5}+k \omega_{6}+\ldots
\end{aligned}
$$

We put these into (19), and equating the coefficients of $\left[\omega_{3} \omega_{5}\right],\left[\omega_{3} \omega_{6}\right],\left[\omega_{1}\left(\omega_{5}\right]\right.$, $\left[\omega_{4} \omega_{6}\right],\left[\xi^{\prime} \omega_{5}\right],\left[\xi^{\prime} \omega_{6}\right],\left[\eta^{\prime} \omega_{5}\right]$, and $\left[\eta^{\prime} \omega_{6}\right]$ to zero we get

$$
\begin{array}{ll}
B k-D z=0, & B l+D w=0 \\
-C l+D w=0, & C k+D z=0 \\
-A l+B w=0, & A k+B z=0 \\
A k-C z=0, & A l+C w=0 .
\end{array}
$$

From each row of these we get

$$
\begin{aligned}
& B\left(k^{2}+l^{2}\right)=D(k z-l w), \quad 0=D(l z+k w) \\
& 0=-D(l z+k w), \quad C\left(k^{2}+l^{2}\right)=-D(k z-l w) \\
& B\left(w^{2}+z^{2}\right)=-A(k z-l w), \quad 0=A(l z+k w) \\
& 0=A(l z+k w), \quad C\left(w^{2}+z^{2}\right)=A(k z-l w) .
\end{aligned}
$$

If $k^{2}+l^{2} \neq 0$ and $w=z=0$, we get by (25) and (26) $B=C=0$, hence by (23) $A=0$. If $w^{2}+z^{2} \neq 0$ and $k=l=0$, we get by (27) (28) and (22) $B=C=D=0$. If $k^{2}+l^{2} \neq 0$ and $w^{2}+z^{2} \neq 0$, we get

$$
\frac{A}{w^{2}+z^{2}}=\frac{B}{-(k z-l w)}=\frac{C}{k z-l w}=\frac{D}{-\left(k^{2}+l^{2}\right)} \text {. }
$$

In any way $A: B: C: D$ is determined when at least one of $k, l, w, z$ is not zero. We treat this case in the first. Then (19) is determined except for a multiplier, and as $d \pi_{1}$ and $d \pi_{2}$ are of this form, we have two cases, namely either $d \pi_{2}=0$ or $d \pi_{1}=m d \pi_{2}\left(d \pi_{2} \neq 0\right)$. If $d \pi_{2}=0$, we get by (17) and (18) $d \pi_{1}=0$, and our space is flat and so this case may be omitted. If $d \pi_{1}=m d \pi_{2}$, we get by (17) and (18)

$$
\begin{aligned}
& {\left[\rho+m \omega_{2}, d \pi_{2}\right]=0,\left[\tau+m \omega_{1}, d \pi_{2}\right]=0} \\
& {\left[\xi+m \omega_{4}, d \pi_{2}\right]=0,\left[\eta+m \omega_{3}, d \pi_{2}\right]=0 .}
\end{aligned}
$$

Now we have by (16)

$$
\begin{aligned}
& \rho+m \omega_{2}=m \omega_{2}+\omega_{3}+w \omega_{5}+z \omega_{6}+\ldots \\
& \tau+m \omega_{1}=m \omega_{1}-\omega_{4}+z \omega_{5}-w \omega_{6}+\ldots
\end{aligned}
$$

which shows that $\rho+m \omega_{2}$ and $\tau+m \omega_{1}$ are independent. Hence by (29)

$$
d \pi_{2}=h\left[\rho+m \omega_{2}, \tau+m \omega_{1}\right]=h\left(\ldots-\left[\omega_{3} \omega_{1}\right]+m\left[\omega_{3} \omega_{1}\right]+\ldots\right) .
$$

Here terms containing $\omega_{1}, \omega_{3}, \omega_{4}$ only are written. Putting this and 


$$
\xi+m \omega_{4}=-\omega_{1}+(p+m) \omega_{4}+k \omega_{5}+l \omega_{6}+\ldots
$$

into (30) we get from the consideration of the term $\left[\omega_{1} \omega_{3} \omega_{1}\right]$ the relation $1-m(p+m)=0$. Hence $m=\left(-p \pm \sqrt{p^{2}+4}\right) / 2$, namely $m$ is a constant. So by (14)

By virtue of $[\omega \Pi]=0$

$$
\Pi=C_{1} d \pi_{1}+C_{2} d \pi_{2}=\left(m C_{1}+C_{2}\right) d \pi_{2} .
$$

$$
\left[\omega\left(m C_{1}+C_{2}\right), d \pi_{2}\right]=0 .
$$

By (31) and (32) each element of the matrix $\omega\left(m C_{1}+C_{2}\right)$ must be a linear combination of $\rho+m \omega_{2}$ and $\tau+m \omega_{1}$, hence the rank of the matrix $m C_{1}+C_{2}$ is two. So by the assumption (13) $C_{1}$ and $C_{2}$ are square matrices of degree 2 anc our Riemann space is of dimension 2. Next we treat the case $k=l=w$ $=z=0$. Then we can assume that in $C_{2}$ all the elements of the first four rows except first four colomns are zero, for in the contrary case we can treat as in the above case already treated, namely the case when one of $k, l, w, z$ is not zero. Thus our space decomposes into a 4-dimensional space and another space, and it is sufficient to consider this 4-dimensional part. For this part $C_{1}$ and $C_{3}$ have components

$$
C_{1}^{(0)}=\left(\begin{array}{cccc}
0 & 1 & 0 & 0 \\
-1 & 0 & 0 & 0 \\
0 & 0 & 0 & 1 \\
0 & 0 & -1 & 0
\end{array}\right), \quad C_{2}^{(0)}=\left(\begin{array}{cccc}
0 & 0 & 1 & 0 \\
0 & 0 & 0 & 1 \\
-1 & 0 & 0 & p \\
0 & -1 & -p & 0
\end{array}\right) .
$$

Hence

$$
\text { det. }\left|m C_{1}^{(0)}+C_{2}^{(0)}\right|=(m(p+m)-1)^{2} .
$$

This is zero for a real number $m=\left(-p \pm \sqrt{p^{2}+4}\right) / 2$. Hence det. $\left|m C_{1}+C_{2}\right|=0$. This contradicts the assumption (13).

Thus we get the result that the Riemann space, for which

$$
\Omega=C_{1} \pi_{1}+C_{2} \pi_{2},
$$

where $C_{1}$ and $C_{2}$ are given by (12) satisfying the relation (13), is two-dimensional.

3. Thus we obtain the result that the Riemann space with two-parametric homogeneons holonomy group decomposes into a certain number of two-dimensional non-flat Riemann spaces and a euclidean space. By lemma 3 the number of these two-dimensional spaces is two, and our proof concludes.

\section{REFERENCES}

[1] S. Sasaki: An alternative proof of Liber's theorem, Proc. Japan Acad, Vol. 27 (1951).

[2] E. Cartan: Leçon sur la géométrie des espaces de Riemann (Paris, Gathier-Villars. 1946).

Mathematical Institute, Nagoya University 